\title{
VEHICLE SCHEDULING IN A HARVEST SEASON
}

\section{Nebojša Gvozdenović ${ }^{1}$, Dejan Brcanov ${ }^{2}$}

*Corresponding author E-mail: nebojsa.gvozdenovic@gmail.com

A R T I C L E IN F O

Original Article

Received: 03 May 2018

Accepted: 01 June 2018

doi:10.5937/ekoPolj1802633G

UDC 629.366:633.63

\section{Keywords:}

vehicle scheduling, harvesting process, sugar beet collection

JEL: C61, Q19, L91

\begin{abstract}
A B S T R A C T
The collection of agricultural goods is a very dynamic process which involves the coordination of hundreds of transport routes and machines with respect the processing capacities at a factory. Numerous fluctuations in the number of transport vehicles, malfunctioning on engaged machines and weather conditions make the process of planning and maximizing the utilization of all resources very difficult. In this paper we present a mathematical model and a heuristic algorithm that in a short period of time finds nearly optimal solutions, which enables a dispatcher to re-plan and update the collection plan according to new constraints.
\end{abstract}

(C) 2018 EA. All rights reserved.

\section{Introduction}

Logistics activities in agriculture range from the simple transportation of goods between facilities, to highly interdependent harvesting process. For many small or medium agricultural associations, most of the transportation is organized on a modest level. As noted by Ali and Van Oudheusden (2009): "most infield harvest operations are still performed without any detailed planning and the efficiency of the process relies mainly on the experience of the workers performing operations." Consequently, the transfer of grain between combines and tractors is delayed and machine performances are reduced. Economical aspects, as well as the accessibility of finances for transportation activities can be found in Sedlak et al. (2016), Fazekaš et al. (2017) and Kontić and Vukasović (2017).

The collection of crops is carried out at the moment when the optimal yield is expected. Harvesting at the "right" moment in practice often implies the engagement of extensive agricultural machinery. According to Gebresenbet and Ljungberg, (2001), the load capacity utilization level of vehicles varies between 10 and $95 \%$. Underused capacities of vehicles testify to the inefficiency of routes. An oversized number of tractors create queues at grain tanks, where experts record waiting times of up to 10 hours.

1 Nebojša Gvozdenović Ph.D., Associate professor, University of Novi Sad, Faculty of Economics in Subotica, Segedinski put no. 9-11, 24000 Subotica, Serbia, Phone: +381 24 628 021, e-mail:nebojsa.gvozdenovic@gmail.com

2 Dejan Brcanov Ph.D., Assistant professor, University of Novi Sad, Faculty of Economics in Subotica, Segedinski put no. 9-11, 24000 Subotica, Serbia, Phone: +381 21485 2923, e-mail:brcanovd@ef.uns.ac.rs, https://orcid.org/0000-0003-4059-5232 
As one of the measures to increase vehicle utilization and minimize the environmental impact, Gebresenbet and Ljungberg, (2001), assert to ,enable vehicle routing and scheduling to fit time windows, with integrated distribution and collection where suitable, as a complement to the drivers'manual route planning". We see the "integrated distribution and collection of goods" as a key to decrease the added value of agricultural products.

A comprehensive study (Fishpool (2016)) on efficiency of sugar beet supply chain addresses main issues of the process: "In summary, the sugar beet supply chain is quite complex and in every area there is huge potential to lose yield or efficiency if not managed effectively. However, there is a great opportunity to use the current resources more efficiently by better planning, monitoring and co-ordination". LópezMilán and Plà-Aragonés (2014) present a mixed integer linear programming model for an agricultural supply chain. The approach was tested on a sugar cane fields at Cuba. Lamsal, Jones and Thomas (2016) focus on problem minimization of number of trucks for the goods transportation from farms to processing plant. Computational tests were performed on sugarcane industry in Lousiana.

This research is focused on transporting agricultural goods from the fields to the factory. Our work was supported by data collected from transporting sugar beets used in sugar production. Although it seems as a rather simple task from a logistic point of view, it is also accompanied by a substantial amount of uncertainty in each part of the operation: a stochastic travel time, an unpredictable queues at loading and/or unloading machines, bad weather conditions, and malfunctions at involved machines. All these difficulties make planning both: arguable and vital for the successful processing of the goods.

The paper is organized as follows. In the next section we present a description of the problem. This is followed by a presentation of the model and algorithm for collection of agricultural goods. Afterwards, we present the results for the proposed algorithm, and in the last section we give some concluding remarks. Logistics activities in agriculture range from the simple transportation of goods between facilities, to highly interdependent harvesting process. For many small or medium agricultural associations, most of the transportation is organized on a modest level. As noted by Ali and Van Oudheusden (2009): "most infield harvest operations are still performed without any detailed planning and the efficiency of the process relies mainly on the experience of the workers performing operations." Consequently, the transfer of grain between combines and tractors is delayed and machine performances are reduced. Economical aspects, as well as the accessibility of finances for transportation activities can be found in Sedlak et al. (2016), Fazekaš et al. (2017) and Kontić and Vukasović (2017).

The collection of crops is carried out at the moment when the optimal yield is expected. Harvesting at the "right" moment in practice often implies the engagement of extensive agricultural machinery. According to Gebresenbet and Ljungberg, (2001), the load capacity utilization level of vehicles varies between 10 and 95\%. Underused capacities of vehicles testify to the inefficiency of routes. An oversized number of tractors create queues at grain tanks, where experts record waiting times of up to 10 hours. 
As one of the measures to increase vehicle utilization and minimize the environmental impact, Gebresenbet and Ljungberg, (2001), assert to ,enable vehicle routing and scheduling to fit time windows, with integrated distribution and collection where suitable, as a complement to the drivers'manual route planning". We see the "integrated distribution and collection of goods" as a key to decrease the added value of agricultural products.

A comprehensive study (Fishpool (2016)) on efficiency of sugar beet supply chain addresses main issues of the process: "In summary, the sugar beet supply chain is quite complex and in every area there is huge potential to lose yield or efficiency if not managed effectively. However, there is a great opportunity to use the current resources more efficiently by better planning, monitoring and co-ordination". LópezMilán and Plà-Aragonés (2014) present a mixed integer linear programming model for an agricultural supply chain. The approach was tested on a sugar cane fields at Cuba. Lamsal, Jones and Thomas (2016) focus on problem minimization of number of trucks for the goods transportation from farms to processing plant. Computational tests were performed on sugarcane industry in Lousiana.

This research is focused on transporting agricultural goods from the fields to the factory. Our work was supported by data collected from transporting sugar beets used in sugar production. Although it seems as a rather simple task from a logistic point of view, it is also accompanied by a substantial amount of uncertainty in each part of the operation: a stochastic travel time, an unpredictable queues at loading and/or unloading machines, bad weather conditions, and malfunctions at involved machines. All these difficulties make planning both: arguable and vital for the successful processing of the goods.

The paper is organized as follows. In the next section we present a description of the problem. This is followed by a presentation of the model and algorithm for collection of agricultural goods. Afterwards, we present the results for the proposed algorithm, and in the last section we give some concluding remarks.

\section{Problem description}

The collection of agricultural goods in peak season requires the efficient use of all resources: loading and unloading machines, transport vehicles and manpower. The underlying processes can be seen as a vehicle scheduling, or as a synchronization of machines involved in acquiring goods. During a typical day in a campaign, a dispatcher coordinates hundreds of vehicle tours, collecting thousands of tons of goods that can be processed according to the factory's capacity. The coordination of all machines with variable input parameters, relying only on dispatcher experience is far from optimal. Therefore, a responsive, robust and scalable system for tracking and planning the collection process is needed.

In a very simplified version, we consider the locations, machines and stocks of agricultural goods that need to be transferred between locations. The locations are factories and fields, and machines are transport vehicles, loaders and off loaders. 
A factory that processes the agricultural goods has a container that defines the upper limit of the goods that can be inside the factory at each moment in time. The lower limit is defined by the inner processes of the factory, and can be seen as a security stocks. During a planning horizon, the level of goods at each moment has to be within the lower and upper limit. Each field has quantity of goods that needs to be transferred to a factory. In this problem, each field and a factory is connected via a path suitable for transport vehicles.

The stocks of goods are transferred to a factory using a fleet of vehicles. The vehicles are homogeneous regarding their capacity, but the number of vehicles fluctuates in respect to the harvesting dynamic of various agricultural goods. Each transferring operation consists of a loading time, a driving time and an unloading time, that are defined by a loading machine, a time matrix and an unloading machine, respectively. The utilization of the vehicles at loading/unloading spots is decided with respect to the formed vehicle queue. The loading and unloading operations are performed by the appropriate machines. During a planning day, a loading machine visits a number of fields, in respect to the order made by a dispatcher. Therefore, the synchronization of loaders and vehicles is essential in order to reduce the non-operating time of vehicles.

The goal of optimization is to minimize the queuing time, with quantities of goods at the factory within the defined limits, given the vehicle fleet, loading/unloading machines and fields with stocks of goods.

Example 1. For the sake of illustration, let us consider a small example including a factory $F$, three fields $L_{1}, L_{2}, L_{3}$ and two loaders 1 and 2 . The stocks at locations require 6 vehicles at location $L_{1}, 5$ vehicles at location $L_{2}$, and 4 vehicles at location $L_{3}$. Loader 1 with an operating time of 6 minutes visits location $L_{1}$, loader 2 visits $L_{2}, L_{3}$ with an operating time of 5 minutes. The unloading time at the factory is 7 minutes.

Figure 1. Illustration of the processes in Example 1.

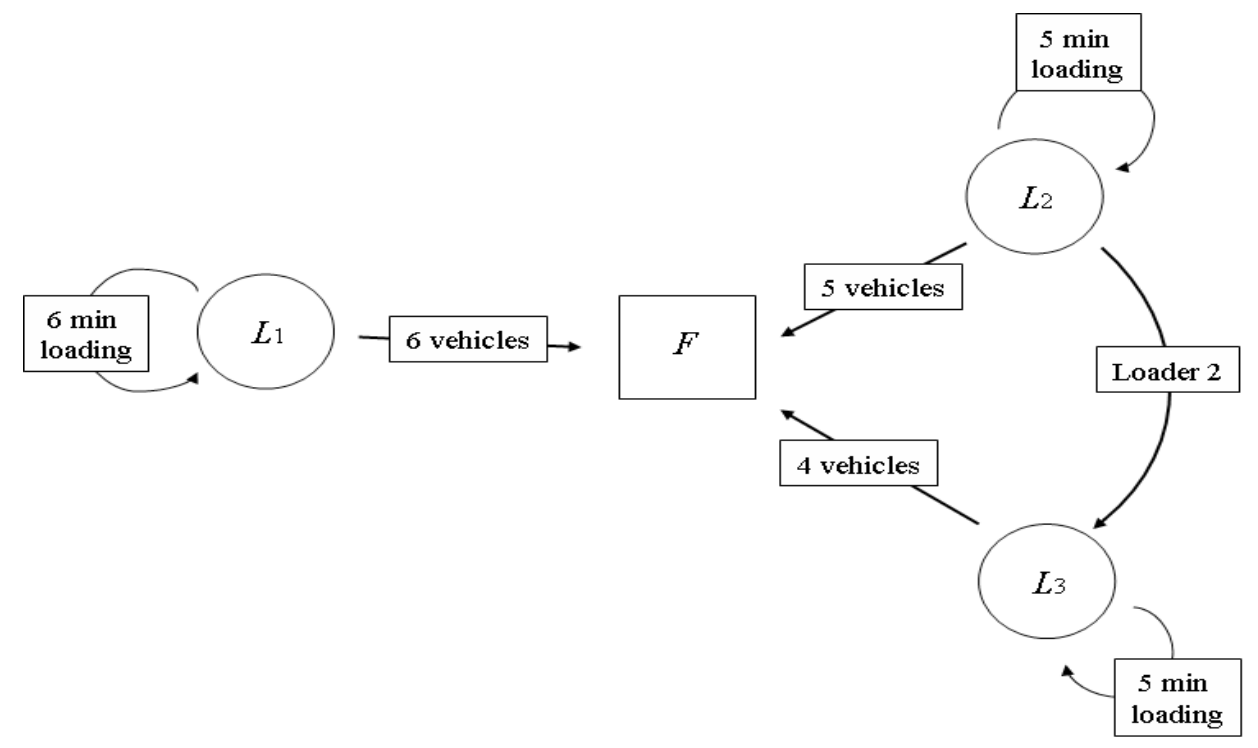




\section{Model}

We use time-span network concept for modeling transport between fields and the factory. A similar approach was used in a study by Bala, Brcanov, Gvozdenović (2016) and Steinzen, Gintner, Suhl, Kliewer (2010). A set of all the locations involved in a planning horizon are denoted with $\Lambda, \Lambda=\left\{L_{0}, L_{1}, L_{2}, \ldots\right\}$, where $L_{0}$ is a factory and $L_{1}, L_{2}, \ldots$ are the fields. Each location is associated with an operating machine $M=\left\{m_{0}, m_{1}, m_{2}, \ldots\right\}$, where $m_{0}$ is an unloading machine and $m_{1}, m_{2}, \ldots$ are the loaders. Note that $m_{1}, m_{2}, \ldots$ are not all necessarily unequal. Each machine from the list has its operating time $t=\left\{t_{0}, t_{1}, t_{2}, \ldots\right\}$. Operating times at each location $L_{i} \in \Lambda$ are used to discretize the timeline at each location $T_{i}=\left\{k \cdot t_{i}, k \in N\right\}$. For any two locations $L_{i}, L_{j} \in \Lambda$ a driving time is denoted with $\operatorname{time}\left(L_{i}, L_{j}\right)$.

Suppose that loading machine $m$ visits $n$ locations $L_{m_{1}}, L_{m_{2}}, \ldots, L_{m_{n}}$, with quantities requiring $v_{m_{1}}, v_{m_{2}}, \ldots, v_{m_{n}}$ vehicles to transport all goods to the factory. And suppose that machine $m$ starts its process at location $L_{m_{i}}$ at time $\alpha_{m_{i}} \in T_{m_{i}}$, and ends at time $\beta_{m_{i}}=\alpha_{m_{i}}+v_{m_{i}} \cdot t_{m_{i}}$.

For each $x \in T_{m_{i}}, \alpha_{m_{i}} \leq x \leq \beta_{m_{i}}$, a ordered triple $\left(L_{m_{i}}, x, y\right)$ where $y=\min \left\{t: t-\left(x+t_{m_{i}}+\operatorname{time}\left(L_{m_{i}}, L_{0}\right)\right)>0, t \in T_{0}\right\}$ represents the actual transport of goods from the field $L_{m_{i}}$ to the factory $L_{0}$.

With the given $\alpha_{m_{0}}$, start time of loading a machine at location $L_{m_{i}}$ for $i>0$, can be calculated using

$\alpha_{m_{i}}=\min \left\{t: t-\left(\beta_{m_{i-1}}+t_{m_{i-1}}+\operatorname{time}\left(L_{m_{i-1}}, L_{m_{i}}\right)\right)>0, t \in T_{m_{i}}\right\}$.

Example 2. For the input defined in example 1with driving times: time $\left(L_{1}, F\right)=40$, time $\left(L_{2}, F\right)=30$, time $\left(L_{3}, F\right)=35$, and time $\left(L_{2}, L_{3}\right)=33$, On Figure 2 we represent the corresponding time-span network. The vertical lines are timelines between the factory and fields and the numbers are the times produced by the loading/unloading times of the machines. 
Figure 2. Timelines for Example 2.

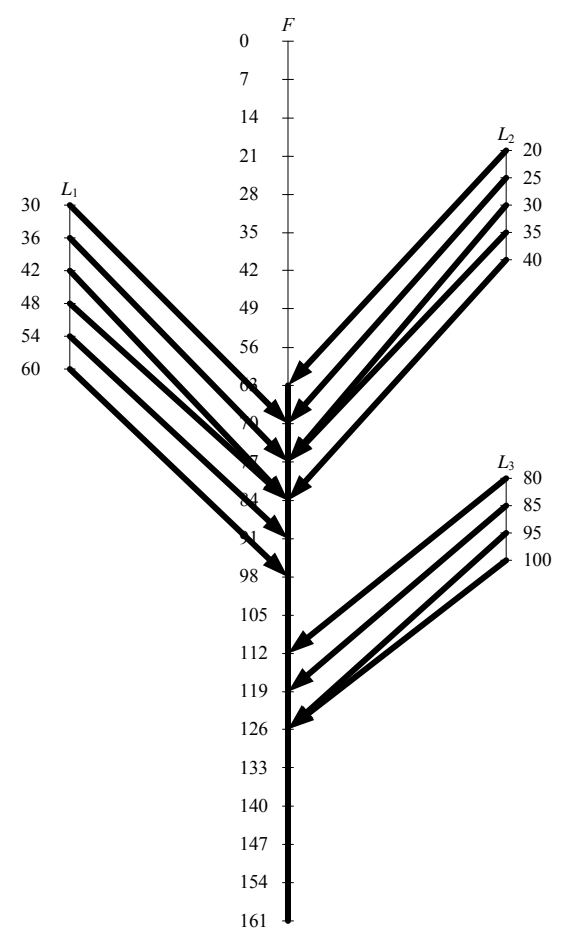

Each transport vehicle has a list of delivery tasks $\left(L_{j}, x_{j}, y_{j}\right)$, which develops into a list of driving tasks $\left(L_{j}, z_{j}, w_{j}\right)$ that include waiting times at fields and the factory. A list is said to be feasible if each task $\left(L_{j}, x_{j}, y_{j}\right)$ and its corresponding driving task $\left(L_{j}, z_{j}, w_{j}\right)$ satisfies

$$
\begin{aligned}
& \text { - } w_{j-1}+\operatorname{time}\left(L_{0}, L_{j}\right) \leq z_{j}, z_{j} \in T_{j} \text { - driving time from the factory to the field, } \\
& \text { - } z_{j} \leq x_{j}, z_{j} \in T_{j} \text { - waiting time in a queue at the loader, } \\
& y_{j} \leq w_{j}, w_{j} \in T_{0} \text { - waiting time in a queue at the factory. }
\end{aligned}
$$

Note that $z_{j}<x_{j}$ if and only if there is a queue at the loader, that is if more than one vehicle has to be loaded at time $z_{j}$, implying $z_{j}+t_{j} \leq x_{j}$ Similarly $y_{j}<w_{j}$ if and only if there is a queue at the factory, that is if more than one vehicle has to be unloaded at time $y_{j}$ or a quantity of goods at time $y_{j}$ at the factory exceeds the upper limit of goods at factory. Note that $y_{j}<w_{j}$ implies $y_{j}+t_{0} \leq w_{j}$.

A set of feasible driving lists constitutes a driving plan. A driving plan is feasible if all input quantities of goods are transferred to the factory while keeping the level of goods at the factory within the limits. 


\section{Solution strategy}

To construct a feasible driving plan, we start from a solution with empty vehicle lists and a dummy list containing all tasks $(L, x, y)$. Clearly, this solution does not correspond to a feasible plan. Neighboring solutions are made via simple transformations of moving a task or swapping two tasks in a current solution. In this way, we consecutively build a solution that corresponds to a feasible driving plan.

Figure 3. Illustration of move and swap transformation

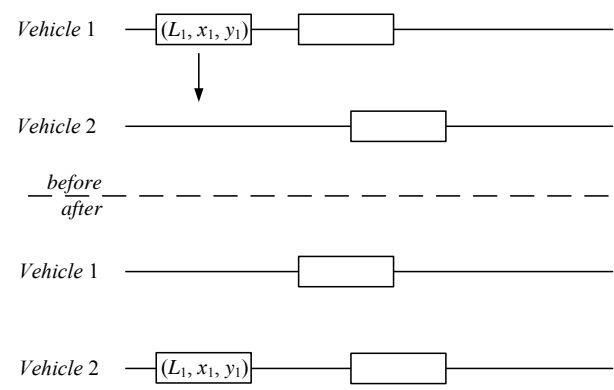

a) move transformation

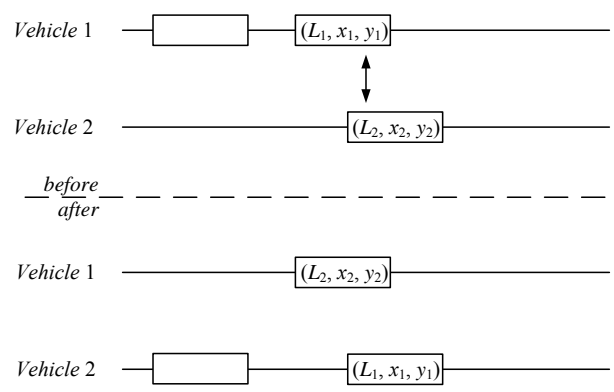

b) swap transformation

To achieve feasibility, we evaluate solutions respecting the not transferred goods, vehicle working time, and additionally power of waiting times at fields and factory.

A searching of the solution space is incorporated within the framework of a simulated annealing heuristic (see Černý (1985) and Kirkpatrick (1983)). For the evaluation value of the current solution $(C)$ and neighboring $(N)$, and the temperature $T$, the algorithm accepts the neighboring solution with probability

$p=\left\{\begin{array}{cc}1, & \text { if } N<C, \\ e^{-\frac{N-C}{T},} & \text { otherwise. }\end{array}\right.$

Temperature $T$ is adjusted after each transformation as $T:=T \cdot q$, where $0<q<1$.

\section{Computational results}

The presented approach has been implemented as an algorithm in $\mathrm{C}++$. The numerous test instances that were considered are real world instances provided by SUNOKO, the largest Serbian producer of sugar. The tests were conducted on a 64-bit PC with 2.70GHz processor and 4GB RAM.

The parameters that define the complexity of a test instance and the corresponding planning scenario are:

- Number of stack locations

- Total quantity of sugar beets that need to be transported to the factory and

- Vehicle capacities (mainly uniform). 
The total number of tasks can be simply calculated from the stack quantities and the vehicle capacities in the case of a uniform fleet of vehicles. We assume that there is enough number of vehicles available. The solution strategy that is used tries to minimize the waiting time of transport vehicles, in order to maximize their re-use and minimize the queue at fields and the factory.

The algorithm was tested on dozens of instances, and the main characteristics of the results are a high degree of stability and minor differences in performance. Table 1 contains results for 10 instances whose number of loading points varies from 2 to 12 .

Table 1. Numerical results

\begin{tabular}{|c|c|c|c|c|c|}
\hline Instance & $\begin{array}{c}\text { Number of } \\
\text { stacks }\end{array}$ & $\begin{array}{c}\text { Total quantity } \\
\text { (tons) }\end{array}$ & $\begin{array}{c}\text { Algorithm } \\
\text { running time } \\
\text { (s) }\end{array}$ & $\begin{array}{c}\text { Number of } \\
\text { tours }\end{array}$ & $\begin{array}{c}\text { Waiting time } \\
\text { in total time } \\
\text { (\%) }\end{array}$ \\
\hline Input2 & 2 & 2858 & 8.042 & 90 & 4.07 \\
\hline Input3 & 3 & 1400 & 7.987 & 45 & 4.22 \\
\hline Input4 & 4 & 2116 & 8.347 & 77 & 2.36 \\
\hline Input5 & 5 & 2300 & 8.04 & 84 & 2.59 \\
\hline Input6 & 6 & 2897 & 8.027 & 93 & 5.05 \\
\hline Input7 & 7 & 2537 & 8.493 & 83 & 5.2 \\
\hline Input8 & 8 & 2884 & 8.068 & 92 & 4.8 \\
\hline Input10 & 10 & 5056 & 8.356 & 161 & 7.96 \\
\hline Input11 & 11 & 2325 & 8.784 & 67 & 7.06 \\
\hline Input12 & 12 & 6455 & 8.973 & 233 & 9.36 \\
\hline
\end{tabular}

\section{Conclusions and future work}

In this research we present a model and a heuristic for the scheduling of transport vehicles in a harvest season. The problem involves hundreds of vehicle tours, and the loading and unloading of machines that have to be synchronized in order to achieve maximal utilization of all resources. The solution strategy is to minimize the waiting time of transport vehicles, in order maximize their re-use and minimize queue at fields and the factory.

The proposed approach was tested on collection of sugar beets, but the framework can be applied to other agricultural goods. The working time of the algorithm is between 8 and 9 seconds, handling up to several hundred of transport tasks and up to 12 fields on a typical day of a campaign. In less than 9 seconds of work time, the approach enables re-routing and adapting route plans to the dynamic changes of the input parameters such as the number of vehicles, suspension of machines due to malfunctions, stochastic driving, and load and unload times.

The next step in building a comprehensive tool is to include the plans of loaders' routes. We expect that such a holistic approach would generate a greater solution space, and consequently improve the synchronization of all machines involved. 


\section{Acknowledgements}

This paper represents a part of the research on the project of the Ministry of Education, Science and Technological Development of the Republic of Serbia, No. 174018, entitled: „Algebraic, logical and combinatorial methods with applications in theoretical computing“.

\section{Conflict of interests}

The authors declare no conflict of interest.

\section{References}

1. Ali, O., Verlinden, B. and Van Oudheusden, D. (2009) Infield logistics planning for crop-harvesting operations, Engineering Optimization, vol. 41, n. 2 https:// doi.org/10.1080/03052150802406540

2. Bala, K., Brcanov, D. and Gvozdenović, N., (2017) Two-echelon location routing synchronized with production schedules and time windows, Central European Journal of Operations Research vol. 25, n. 3, p. 525-543.

3. Černý, V., (1985) Thermodynamical approach to the traveling salesman problem: An efficient simulation algorithm, Journal of Optimization Theory and Applications vol. 45, p. 41-51.

4. Fazekaš, T., Bobera, D., Ćirić, Z. (2017) Ecologically and Economiccaly Sustainable

5. Agricultural Transportation Based on Advanced Information Technologies, Journal Economics of Agriculture, Vol. 64 (2) p. 405-860.

6. Fishpool, P., Maximising the efficiency of the UK Sugar Beet supply chain (2016) A Nuffield (UK) Farming Scholarships Trust Report, http:// nuffieldinternational.org/live/Report/UK/2015/paul-fishpool.

7. Gebresenbet, G. and Ljungberg, D., (2001) IT-Information Technology and the Human Interface, Journal of Agricultural Engineering Research, vol. $80 \mathrm{n}$. 4, p. 329-342.

8. Kirkpatrick, S., Gelatt Jr, C. D. and Vecchi, M. P. (1983) Optimization by Simulated Annealing, Science vol. 220 p. 671-680. doi:10.1126/ science.220.4598.671

9. Kontić, Lj., Vukasović, D., The Impact of Grains Transportation Revenues on Total Revenue: The Case of Serbia, Journal Economics of Agriculture (2017) Vol. 64, (2) p. 483-495.

10. Lamsal, K., Jones, P.C., Thomas, B.W., Harvest logistics in agricultural systems with multiple, independent producers and no on-farm storage, Computers \& Industrial Engineering (2016) Vol. 91, 129-138 
11. López-Milán, E. \& Plà-Aragonés, L.M., A decision support system to manage the supply chain of sugar cane, Annals of Operations Research (2014) Vol. 219 (1) 285-297. https://doi.org/10.1007/s10479-013-1361-0

12. Sedlak, O., Jovin, S., Pejanović, R., Ćirić, Z., Eremić Đođić, J. (2016) Access to Finance for Micro, Small, and Medium Business Units in Serbian Agribusiness, Journal Economics of Agriculture (2016) Vol. 63, (4) p. 1113-1484.

13. Steinzen, I., Gintner, V., Suhl, L. and Kliewer, N., (2010) A time-space network approach for the integrated vehicle and crew-scheduling problem with multiple depots, Transportation Science vol.44, p. 367-382. 